\title{
Bispectral Photometric Stereo based on Fluorescence
}

\author{
Imari Sato \\ National Institute of Informatics \\ Tokyo, Japan \\ imarikenii.ac.jp
}

\begin{abstract}
We propose a novel technique called bispectral photometric stereo that makes effective use of fluorescence for shape reconstruction. Fluorescence is a common phenomenon occurring in many objects from natural gems and corals, to fluorescent dyes used in clothing. One of the important characteristics of fluorescence is its wavelengthshifting behavior: fluorescent materials absorb light at a certain wavelength and then reemit it at longer wavelengths. Due to the complexity of its emission process, fluorescence tends to be excluded from most algorithms in computer vision and image processing. In this paper, we show that there is a strong similarity between fluorescence and ideal diffuse reflection and that fluorescence can provide distinct clues on how to estimate an object's shape. Moreover, fluorescence's wavelength-shifting property enables us to estimate the shape of an object by applying photometric stereo to emission-only images without suffering from specular reflection. This is the significant advantage of the fluorescence-based method over previous methods based on reflection.
\end{abstract}

\section{Introduction}

Fluorescence emission is a common phenomenon occurring in many objects from natural gems and corals, to fluorescent dyes used for clothing; even laundry detergent contains fluorescence dyes to brighten/whiten clothing. Through intensive studies on color constancy algorithms, Barnard concluded that fluorescent surfaces are common and present in $20 \%$ of randomly constructed scenes [2]. What makes fluorescence different from ordinary reflection is the transfer of energy from one wavelength to another. More specifically, fluorescent materials absorb light at a certain wavelength and then reemit it at other wavelengths after about $10^{-8}$ seconds, whereas ordinary reflective components reflect light at the same wavelength as incident.

Due to the complexity of its emission process, fluorescence tends to be excluded from most algorithms in

\author{
Takahiro Okabe, Yoichi Sato \\ University of Tokyo \\ Tokyo, Japan \\ takahiro,ysato@is.u-tokyo.ac.jp
}
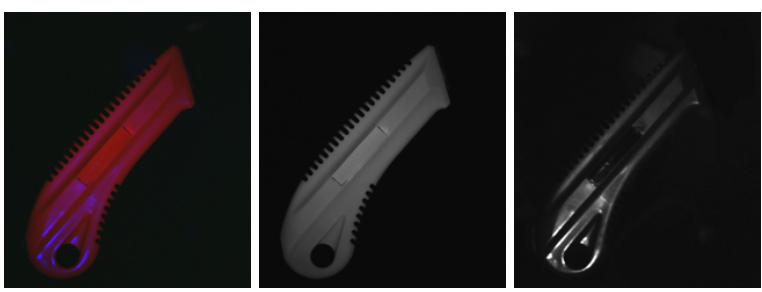

Figure 1. Reflective and fluorescent components separated through bispectral measurement: image of a cutter under near UV light (left), fluorescent-only image observed in the red channel (middle), and reflective-component-only images observed in the blue channel of the camera (right).

computer vision and image processing. In this paper, we show that fluorescence has the potential of providing clues for estimating an object's surface normals and present a novel photometric stereo approach based on fluorescence observed by a technique called bispectral measurement wherein the incident and received wavelengths are different. The reflective component is seen only when the incident and outgoing wavelengths are the same, and this allows us to obtain emission-only images by simply setting a longer wavelength than the incident one as the received wavelength.

The classical photometric stereo approach [27] recovers the shape of an object having Lambertian reflectance from multiple images taken of it under known light sources. Although photometric stereo has been intensively studied to cope with objects having complex appearances and/or deal with illuminations from unknown directions, most approaches focus on the reflections from the object's surface, and no attention has been paid to the re-radiation (emission) of an object's fluorescent materials. In this work, we propose to use fluorescence from an object containing both non-fluorescent and fluorescent materials for shape reconstruction. In fact, there is a strong similarity between the fluorescence emission and the ideal diffuse reflection.

Wilkie et al. used this fact for modeling fluorescent emissions as diffuse reflections with a wavelength-shifting property, i.e., a diffuse surface that reflects light at a wavelength different from the incident one [25]. Their development mo- 
tivated us to create a new method for shape reconstruction based on fluorescence observed by bispectral measurement. As far as we know, this is the first attempt to use fluorescence from an object for estimating its surface normals.

Fluorescence measured bispectrally provides the following advantages for photometric stereo. First, by simply having different incident and received wavelengths, we can avoid observing reflections including troublesome specular reflection and utilize emission-only images for shape reconstruction. For instance, Figure 1 shows the reflective and fluorescent components of an object separated through bispectral measurement. We see that fluorescent-only image observed under near UV light in the red channel of a camera is free from specularity (Figure 1 middle). Secondly, interreflections caused by fluorescence emissions are much less than those caused by diffuse reflections: a fluorescent material will not be excited much by its own emission. Specular reflection and interreflection often decrease the accuracy of shape reconstruction. The use of emission-only images has significant advantages over previous methods based on reflection. The contributions of our paper are

- presenting a bispectal radiance equation that describes the brightness changes of an object containing both non-fluorescent and fluorescent components under different lighting directions,

- using bispectral measurement for separating reflective and fluorescent components, and

- presenting a new approach for photometric stereo based on fluorescence emission.

The rest of the paper is structured as follows. Section 2 summarizes research on photometric stereo and on color appearance and rendering algorithms for fluorescent surfaces. Section 3 describes the characteristics of fluorescence and presents the bispectral radiance equation that represents the appearance of a composite object containing both ordinary reflective and fluorescent components. We discuss the advantages of using the fluorescence captured by bispectral measurements in Section 4 and then present our bispectral photometric stereo method in Section 5. Experiments on real images of composite objects are presented in Section 6 . In the conclusion, we discuss the future directions of our research.

\section{Related Work}

Shape recovery using photometric stereo. The classical photometric stereo approach [27] recovers the shape of an object having Lambertian reflectance from multiple images of it taken under known light sources. Later, this approach was extended to include a broader range of circumstances such as non-Lambertian surfaces[16, 8, 22, 11, 29, 4] (for a good overview, see [26]), and uncalibrated photometric stereo [13, 6, 5] (for the cases of unknown light sources). Some researchers studied the effect of interreflection between object surfaces for shape reconstruction $[20,7,21,18]$.

While many techniques have been proposed for solving different problems, almost all of them exploit only reflections from the object's surface. Our approach differs from these approaches in that we do not use reflection at all but rather utilize fluorescent emissions (reradiation by fluorescent materials) observed bispectrally.

Fluorescence. The apparent color of non-fluorescent surfaces has been intensively studied in color-related computer vision algorithms. Barnard compared various color constancy algorithms with a large set of test images that included some images of fluorescent objects [3]. The evaluated algorithms however treated fluorescence as being composed of ordinary reflective components. Later, Barnard proposed ways to improve color constancy algorithms so that they could deal with spectral data of several fluorescent materials [2]. There have also been studies on separating the reflective and fluorescent components observed in composite objects $[19,28]$. Alterman et al. separated the appearances of individual fluorescent dyes to analyze underlying mixture of materials by unmixing multiplexed images [1].

Researchers in the field of computer graphics have also realized that assuming all objects are non-fluorescent greatly limits the quality of the rendered objects because many objects in the real world exhibit fluorescence. Glassner first incorporated the wavelength-shifting property of fluorescence into Kajiya's rendering equation [12]. Johnson and Fairchild provided brief explanations of fluorescence phenomena and extended spectral rendering algorithms to take fluorescence into account [17].

Bispectral methods are often used for modeling fluorescence emissions in the field of fluorometry, in which the spectral distribution of the fluorescence is measured as a function of the incident and outgoing wavelengths: Donaldson's re-radiation matrices are used for representing fluorescent spectral radiation factors whose elements are defined by the incident and outgoing wavelengths [9]. Pure fluorescent material has no directional characteristics [12], and fluorescence radiance factors are often captured with a fixed measurement geometry, e.g., $0^{\circ} / 45^{\circ}$ or $0^{\circ} / 10^{\circ}$ for incident and received light and vice versa.

Later, Wilkie et al. showed that even a fluorescent diffuse sheet contains a small but significant amount of specularity that produces some directional dependence in its appearance [25], and they represented its appearance as a combination of fluorescent and non-fluorescent semi-gloss specular components. In particualr, they represented fluorescence as a diffuse reflection with a wavelength-shifting property, i.e., a diffuse surface that reflects a different wavelength 


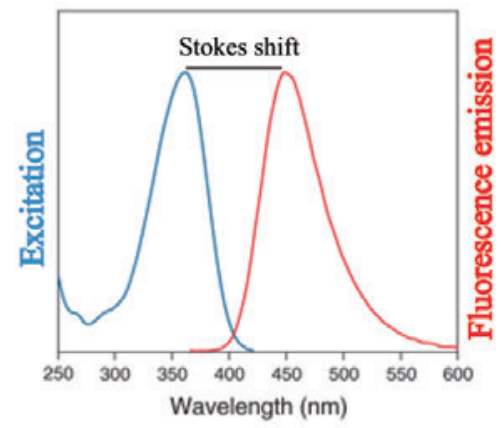

Figure 2. Excitation and emission spectra of a fluorescent material

from the incident one. More recently, Hullin et al. proposed a more general and efficient way to model the appearance of objects containing both non-fluorescent and fluorescent materials with bispectral bidirectional reflectance and reradiation distribution functions (BRRDF) [15]. Their method resulted in a significant improvement in fluorescent object models.

Regarding shape reconstruction, Hullin et al. introduced a fluorescent immersion scanning system where an object was embedded into a fluorescent liquid [14]. In this system, light paths going through the fluorescent liquid become visible, and their incidence with the object's surface can then be analyzed. Our approach is different in that it directly uses the fluorescence from an object and it does not treat fluorescent materials as a light transfer medium.

\section{Bispectral Models for Reflectance and Rera- diation}

After decades of studies, fluorescence phenomena are now able to be described with quantum theory [10, 24]: fluorescent materials always emit light at longer wavelengths than the absorbed light and the emission spectra always have the same frequency distribution (shape) regardless of the spectra of incident light. Namely, fluorescence shows a constant color (chromaticity) that is not affected by the color of the illumination [28].

The emissions of fluorescent materials are characterized by their excitation and emission spectra. The excitation spectra show how much energy from the illuminant is absorbed at each wavelength, and the emission spectra show the spectral distribution of the emitted light from the fluorescent surfaces. Figure 2 shows the normalized excitation and emission spectra of one fluorescent material. ${ }^{1}$ The difference in wavelength between the positions of the maxima of the excitation and emission spectra is known as Stokes shift.

\footnotetext{
${ }^{1}$ The spectral power distribution is normalized so that the minimum intensity is 0 and the maximum intensity is 1.0 .
}
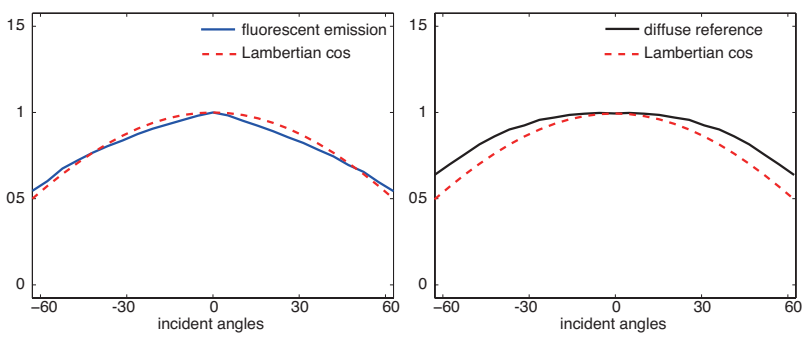

Figure 3. Radiance changes due to different lighting directions: fluorescent emission (left) and diffuse surface (right)

\subsection{Bispectral Model}

To model the appearance of an object with ordinary reflective and fluorescent components, we need to consider the transfer of energy from one wavelength to another. Recently, Hullin et al. devised a Bispectral Bidirectional Reflectance and Reradiation Distribution Function (bispectral BRRDF) $r\left(\boldsymbol{\omega}_{i}, \boldsymbol{\omega}_{o}, \lambda_{i}, \lambda_{o}\right)$ that describes the angularly dependent reflectance for any pair of incoming and outgoing wavelengths [15]. In this function, $\boldsymbol{\omega}_{i}$ and $\boldsymbol{\omega}_{o}$ are the incident and reflection directions with respect to the surface normal of the object, and $\lambda_{i}$ and $\lambda_{o}$ represent the incident and outgoing wavelengths. The observed spectrum of a surface based on the bispectral BRRDF is

$$
E\left(\boldsymbol{\omega}_{i}, \boldsymbol{\omega}_{o}, \lambda_{i}, \lambda_{o}\right)=r\left(\boldsymbol{\omega}_{i}, \boldsymbol{\omega}_{o}, \lambda_{i}, \lambda_{o}\right) L\left(\boldsymbol{\omega}_{i}, \lambda_{i}\right) \cos \theta_{i},
$$

where $L\left(\boldsymbol{\omega}_{i}, \lambda_{i}\right)$ is the illumination coming from the direction $\boldsymbol{\omega}_{i}$ at wavelength $\lambda_{i}$, and $\theta_{i}$ is the angle between the surface normal and the light-source direction $\boldsymbol{\omega}_{i}$.

Classic photometric stereo requires an object to have a reflectance that is independent of the incident and outgoing directions. One such example is an ideal diffuse surface whose brightness can be described as

$$
E\left(\boldsymbol{\omega}_{i}, \lambda\right)=\rho(\lambda) L\left(\boldsymbol{\omega}_{i}, \lambda\right) \cos \theta_{i},
$$

where $\lambda_{i}=\lambda_{o}=\lambda$ and $r\left(\boldsymbol{\omega}_{i}, \boldsymbol{\omega}_{o}, \lambda_{i}, \lambda_{o}\right)$ in Equation 1 is simplified to the albedo of the object $\rho(\lambda)$. It follows from this that, given the different directions of light with unit radiance at the wavelength $\lambda$, the brightness of a diffuse surface changes proportionally to $\cos \theta_{i}$. Classic photometric stereo makes use of this fact for estimating surface normals.

It is known that fluorescence has no directional character and is radiated uniformly in all directions [12]. A fluorescent emission behaves like it is from an ideal diffuse surface except for the fact that it absorbs and re-emits light at a different wavelength from the incident one [25]. Accordingly, it is found that the re-radiation property of fluorescence can be described by a directionally independent function $\mu\left(\lambda_{i}, \lambda_{o}\right)$, and the emission from a fluorescent material illuminated by light $L\left(\boldsymbol{\omega}_{i}, \lambda_{i}\right)$ can computed as

$$
E\left(\boldsymbol{\omega}_{i}, \lambda_{i}, \lambda_{o}\right)=\mu\left(\lambda_{i}, \lambda_{o}\right) L\left(\boldsymbol{\omega}_{i}, \lambda_{i}\right) \cos \theta_{i}
$$


Since fluorescent materials always emit light at longer wavelength than that of the absorbed light, $\mu\left(\lambda_{i}, \lambda_{o}\right) \simeq 0$ for $\lambda_{i} \geq \lambda_{o}$. Given fixed $\lambda_{i}$ and $\lambda_{o}$ for $\lambda_{i}<\lambda_{o}$, the observed emission $E\left(\boldsymbol{\omega}_{i}, \lambda_{i}, \lambda_{o}\right)$ becomes proportional to $\cos \theta_{i}$. We observed fluorescence from several objects under different lighting directions and verified that the brightness of the fluorescence (blue solid as shown in Figure 3 left) could be well approximated by a Lambertian cosine law (red dotted line).We also verified that fluorescence is radiated uniformly in all directions, as described by Glassner [12]. It is known that the Lambertian model is sometimes not adequate to represent real-world rough surfaces such as concrete, plaster, sand, and so on [23]. For reference, we measured the radiance changes in a white diffuse reflectance target. We see a deviation from the Lambertian model that is due to surface roughness (Figure 3 right).

Let us go back to the original radiance equation and consider the appearance of a composite object containing both ordinary reflective and fluorescent components. Equation 1 can be rewritten as the sum of the brightnesses of the reflective and fluorescent components:

$$
\begin{aligned}
E\left(\boldsymbol{\omega}_{i}, \boldsymbol{\omega}_{o}, \lambda_{i}, \lambda_{o}\right) & =\left[f\left(\boldsymbol{\omega}_{i}, \boldsymbol{\omega}_{o}, \lambda_{o}\right) \delta\left(\lambda_{i}-\lambda_{o}\right)\right. \\
& \left.+\mu\left(\lambda_{i}, \lambda_{o}\right)\right] L\left(\boldsymbol{\omega}_{i}, \lambda_{i}\right) \cos \theta_{i} .
\end{aligned}
$$

Here, the reflective component (the first term) is characterized by a bidirectional reflectance distribution function BRDF $f\left(\boldsymbol{\omega}_{i}, \boldsymbol{\omega}_{o}, \lambda_{o}\right)$. A delta function $\delta\left(\lambda_{i}-\lambda_{o}\right)$ is associated with it because an ordinary reflective component reflects light at the same wavelength as $\lambda_{i}$. In other words, we do not observe any brightness when $\lambda_{i} \neq \lambda_{o}$. From Equation 3, we can see that some combinations of incident and receiving wavelengths reveal certain properties about the object.

Reflective component only: $\lambda_{i}=\lambda_{o}$. When the incident and received light have the same wavelength, i.e. $\lambda_{i}=\lambda_{o}$, almost no contribution from fluorescent component is observed because $\mu\left(\lambda_{i}, \lambda_{o}\right) \simeq 0$ for $\lambda_{i} \geq \lambda_{o}$. What we observe is only the contribution from the non-fluorescent component.

Fluorescent components only: $\lambda_{i}<\lambda_{o}$. When the wavelengths are different such that $\lambda_{i}<\lambda_{o}$, there is no contribution from the reflective component because $\delta\left(\lambda_{i}-\lambda_{o}\right)=0$. Therefore, only the emission from fluorescent component is seen.

\section{Advantages of bispectral measurements for shape reconstruction}

In the previous section, we saw that the wavelengthshifting properties of the fluorescence emission are useful for analyzing different properties of an object having ordinary reflective and fluorescent component emissions. In particular the ability to adjust the incident and observing wavelengths provides the following advantages for shape reconstruction.

\subsection{Specular free image}

Specular reflection belongs to the reflective component and is seen when the incident and outgoing wavelengths are the same. In other words, we can avoid observing specular reflection by simply setting a different wavelength from the incident one as $\lambda_{o}$. Figure1 shows an example of specularfree images observed under near UV light in the red channel of a CCD camera. ${ }^{2}$ The object contains non-fluorescent and fluorescent components. Simply having the wavelengths so that $\lambda_{i}<\lambda_{o}$ provides an image of fluorescent component only (Figure 1 middle). This enables us to use specular-free emission images for shape reconstruction. In this example, this object contains a few diffuse components, and thus, the image obtained for $\lambda_{i}=\lambda_{o}$ in the blue channel clearly shows specular reflection (Figure1 right).

\subsection{Less interreflection between fluorescent sur- faces}

For a concave object, interreflection between object surfaces often decreases the accuracy of the reconstructed shape. The wavelength-shifting property of fluorescent material lessens the influence of interreflection. In accordance with the Stokes shift, a fluorescent material will not be excited much by its own emissions because there is not much overlap between its excitation and the emission spectra (For instance, see Figure 2). Accordingly, interreflections caused by fluorescence emissions are much less that those caused by diffuse reflections for scenes with a single material.

It should be noted that the emitted light from fluorescence materials have a chance of becoming secondary light and being reflected by other surfaces. This happens only when other surfaces have spectral reflectances within the range of its emission spectra. In our experiments, most objects consisted of a single material and contained different colors of fluorescent and reflective components, and we did not observe such phenomena. In the case of scenes with different materials, however, the secondary light might be reflected by other surfaces. We will investigate the interaction between fluorescence and reflection in the future.

\section{Bispectral Photometric Stereo}

Photometric stereo estimates the surface normal of an object by observing the object under different lighting conditions. Given irradiance $E\left(\boldsymbol{\omega}_{k}, \lambda\right)(k=1,2, \ldots, K)$ at a

\footnotetext{
${ }^{2}$ In Section 5.1 we describes the conditions to observe fluorescence using an ordinary color CCD camera and a light source.
} 
point on a diffuse surface, we obtain a set of linear equations of the form

$$
\begin{aligned}
E\left(\boldsymbol{\omega}_{k}, \lambda\right) & =\rho(\lambda) L\left(\boldsymbol{\omega}_{k}, \lambda\right) \cos \theta_{k} \text { or } \\
e_{k} & =\rho \boldsymbol{n} \cdot \boldsymbol{l}_{k},
\end{aligned}
$$

where $\boldsymbol{n}$ is a surface normal of this point and $\boldsymbol{l}_{k}$ is the direction of the $k$ th light source. If the light-source directions are known, we can recover $\rho \boldsymbol{n}$ by using linear least squares.

Let us extend the classic photometric stereo approach to the case of bispectral measurements where the incident and receiving wavelengths are different: $\lambda_{i} \neq \lambda_{o}$. As described in Section 3, when the received wavelength is longer $\left(\lambda_{i}<\lambda_{o}\right)$, we observe only the contribution from the fluorescence materials, whose appearance is described by Equation 2. Given a fixed $\lambda_{i}$ and $\lambda_{o}$ for $\lambda_{i}<\lambda_{o}$, the observed emission $E\left(\boldsymbol{\omega}_{k}, \lambda_{i}, \lambda_{o}\right)$ becomes proportional to the angle between the surface normal and the light source direction and has a form similar to that of the diffuse light:

$$
\begin{aligned}
E\left(\boldsymbol{\omega}_{\boldsymbol{k}}, \lambda_{i}, \lambda_{o}\right) & =\mu\left(\lambda_{i}, \lambda_{o}\right) L\left(\boldsymbol{\omega}_{k}, \lambda_{i}\right) \cos \theta_{k} \text { or } \\
e_{k} & =\mu \boldsymbol{n} \cdot \boldsymbol{l}_{k},
\end{aligned}
$$

from which we can recover $\mu \boldsymbol{n}$ by using least squares. These equations are well-conditioned as long as the (three or more) light source directions $\boldsymbol{l}_{k}$ are linearly independent.

\subsection{Observing emission-only images using RGB camera and colored light source}

In practice, it would be convenient if we could observe the fluorescent component by using an ordinary color CCD camera and a general light source. From Equation 3, the appearance of a composite object under illumination at all wavelengths is

$$
\begin{array}{r}
E\left(\boldsymbol{\omega}_{i}, \boldsymbol{\omega}_{o}, \lambda_{i}, \lambda_{o}\right)=\left[f\left(\boldsymbol{\omega}_{i}, \boldsymbol{\omega}_{o}, \lambda_{o}\right) L\left(\boldsymbol{\omega}_{i}, \lambda_{o}\right)\right. \\
\left.+\int \mu\left(\lambda_{i}, \lambda_{o}\right) L\left(\boldsymbol{\omega}_{i}, \lambda_{i}\right) \mathrm{d} \lambda_{i}\right] \cos \theta_{i} .
\end{array}
$$

Here, the fluorescent component has the wavelengthshifting property, and thus, the overall observed spectrum needs to be computed by summing up $\mu\left(\lambda_{i}, \lambda_{o}\right) L\left(\boldsymbol{\omega}_{i}, \lambda_{i}\right)$ for all wavelengths $\lambda_{i}$. In contrast, only the reflection due to the illumination at the wavelength $\lambda_{o}$, i.e. $L\left(\boldsymbol{\omega}_{i}, \lambda_{o}\right)$, needs to be considered for the non-fluorescent component.

From Equation 4, we can see that the following conditions need to be satisfied to obtain emission-only images. First, the illuminant should have no energy at the outgoing wavelength. i.e. $L\left(\boldsymbol{\omega}_{i}, \lambda_{o}\right)=0$. This results in avoiding observing the reflective component. Secondly, the fluorescent material should absorb some energy from the light and emit light in the wavelength $\lambda_{o}$ : $\int \mu\left(\lambda_{i}, \lambda_{o}\right) L\left(\boldsymbol{\omega}_{i}, \lambda_{i}\right) \mathrm{d} \lambda_{i}>0$.

Next, let us consider the case where the scene is observed by a color camera. If we have a camera with three channels
( $\mathrm{R}, \mathrm{G}$, and $\mathrm{B}$ ), the brightness of a surface point of the object for each channel is

$$
\begin{aligned}
& P_{q}=\int c_{q}\left(\lambda_{o}\right)\left[f\left(\boldsymbol{\omega}_{i}, \boldsymbol{\omega}_{o}, \lambda_{o}\right) L\left(\boldsymbol{\omega}_{i}, \lambda_{o}\right)\right. \\
& \left.+\int \mu\left(\lambda_{i}, \lambda_{o}\right) L\left(\boldsymbol{\omega}_{i}, \lambda_{i}\right) \mathrm{d} \lambda_{i}\right] \mathrm{d} \lambda_{o} \cos \theta_{i},
\end{aligned}
$$

where $c_{q}(q=r, g, b)$ are the camera spectral sensitivities for each channel. Similar to the previous case, the following conditions need to be satisfied to observe emission-only images. For simplicity, let us consider the case where we observe the scene in the red channel of the camera $c_{r}$ here.

First, the illuminant should have no energy within the effective range of $c_{r}$. i.e. $\int c_{r}\left(\lambda_{o}\right) L\left(\boldsymbol{\omega}_{i}, \lambda_{o}\right) \mathrm{d} \lambda_{o}=0$. If there is no overlap between the illumination spectra and the effective range of $c_{r}\left(\lambda_{o}\right)$, the observed brightness in the red color channel does not have reflective components. ${ }^{3}$

Secondly, the fluorescent material should absorb some energy from light and emit light in the effective range of $c_{r}\left(\lambda_{o}\right): \int c_{r}\left(\lambda_{o}\right)\left[\int \mu\left(\lambda_{i}, \lambda_{o}\right) L\left(\boldsymbol{\omega}_{i}, \lambda_{i}\right) \mathrm{d} \lambda_{i}\right] \mathrm{d} \lambda_{o}>0$. Namely, the excitation spectra of the fluorescent material needs to overlap with the illumination spectra, and its emission spectra needs to overlap with the effective range of $c_{r}\left(\lambda_{o}\right)$. In our experiments, we illuminated objects with a blue halogen lamp or LED UV light whose spectra do not overlap with the effective range of the spectral sensitivities for the green and red channels of a camera.

\section{Results and Analysis}

We tested our technique on images of real objects containing both fluorescent and reflective components. The objects used in our experiment naturally exhibit fluorescence except the ceramic dish example. To obtain their emissiononly images, we illuminated objects with LED UV light (for ceramic dish example) or a blue halogen lamp (for other examples) and captured object images in the red or green channels of a CCD camera (SONY DXC-9000). For each object, $15 \sim 30$ images were captured with the object illuminated from different light source directions. The light source was about $1 \mathrm{~m}$ away from the object, which was $3 \sim 8 \mathrm{~cm}$ in diameter.

We first evaluated the accuracy of our technique on a diffuse fluorescent sphere. Figure 4(a) (left) shows the appearance of the sphere under a white halogen lamp. Using UV light, we checked that the color of its fluorescent component was green and its emission spectra fitted within the range of the color sensitivity in the green channel of this camera. The sphere also contained ordinary reflective components at longer wavelengths corresponding to red. This allowed us to separately observe their contributions by using different combinations of light and color channels of the

\footnotetext{
${ }^{3}$ More intuitively, this means that we do not observe any reflection in the red channel under blue illumination.
} 

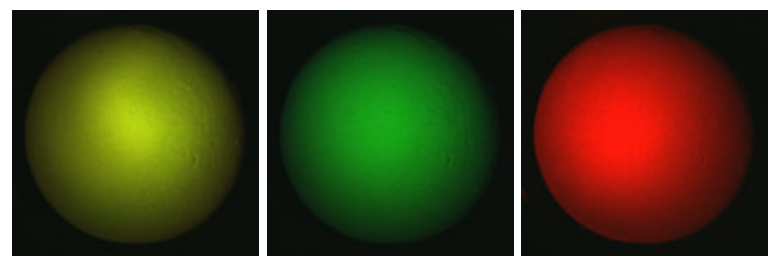

(a) Images of object under white (for reference), blue light (for extracting fluorescence emission), and red light (for observing diffuse reflection).

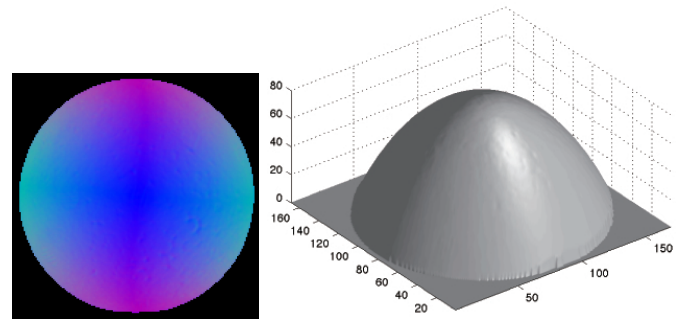

(b) Shape recovered from reflection images.
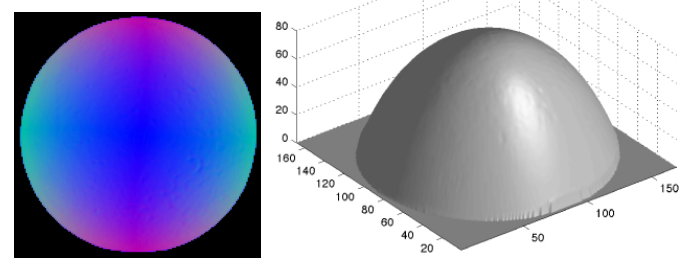

(c) Shape recovered from emission images.

Figure 4. Fluorescent diffuse sphere containing both fluorescent and reflective components.

camera. Images captured under blue light (Figure 4(a) middle) in the green channel show fluorescence only (emission images), and images captured under red light (Figure 4(a) right) in the red channel show diffuse reflection (reflection images).

Figures 4(b) and 4(c) show the estimated surface normals and shapes of the sphere from reflection images and emission images respectively: the estimated surface normals are stored directly in the RGB values of the surface normal map images. The absolute values of the $x, y$, and $z$ coordinates are shown in red, green, and blue, respectively. ${ }^{4}$

Compared with the shape obtained from reflection images based on photometric stereo (Figure 4(b)), we can clearly see that our bispectral photometric stereo method can estimate the surface normals and shape of the object with equivalent accuracy (Figure 4(c)). The rootmean-square error between the surface normal directions estimated from fluorescence emission and the ground-truth was 2.0 degrees, and the error between those estimated

\footnotetext{
${ }^{4} x$ and $y$ axes correspond to the horizontal and vertical axis of the image plane, and the $z$ axis is defined to be perpendicular to the image plane.
}
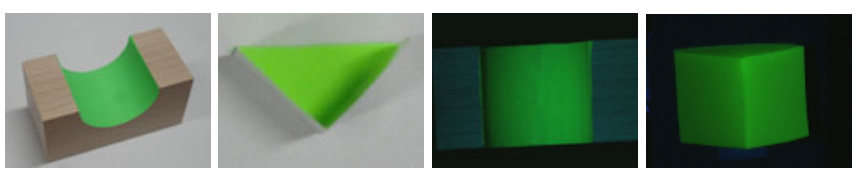

(a) images of objects under natural lighting (for reference) and input images of objects under blue light.
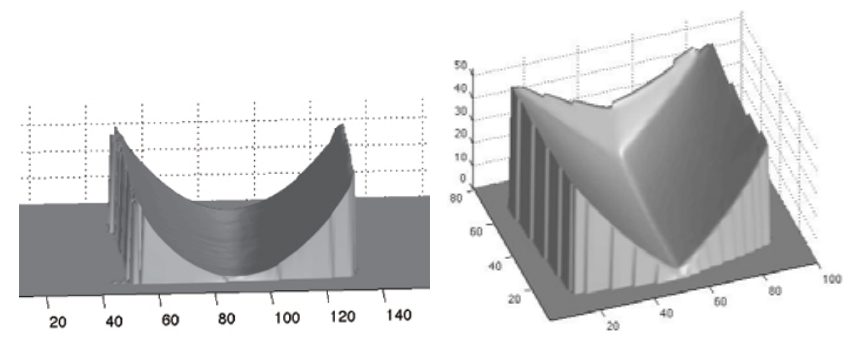

(b) Recovered shape for bridge-shaped and L-shaped objects.

Figure 5. Experimental results for concave objects.

from diffuse reflections and the ground-truth was 3.4 degrees. This shows that the photometric stereo approach based on fluorescence emission performs better than that based on diffuse reflection in this experiment.

Concave Objects. We also tested our technique on concave objects covered with different fluorescent materials. Figure 5(a) shows the objects used in this experiment: a bridgeshaped wooden object painted fluorescent green and a Lshaped green fluorescent sheet. Figures 5(b) show the estimated shapes. In this figure, we can see that our method could estimate their shapes regardless of their concavity. This demonstrates the robustness of our method against interreflections between object surfaces. As described before, a fluorescent material will not be excited that much by its own emission, and this property helps to make the estimated shapes accurate even for concave objects.

Object with specular highlights . Our method is also effective on objects with specular reflection. The first test object was a key holder whose fluorescent component shows green color. Figure 6(a) shows the appearance of this object under blue light. We can see its fluorescent and reflective components in the green and blue channels of this image. In Figure 6(a) (right), the image of the reflective component shows a significant amount of specular reflection, while that of the fluorescent component (Figure 6(a) middle) is free from specularity. This enabled us to reliably estimate the shape of the object by applying photometric stereo to its fluorescence images without suffering from specular reflection. The recovered shape is shown in Figures 6(b).

This object contained ordinary reflective components at longer wavelengths corresponding to red. For comparison, the shape of the object was estimated using images contain- 

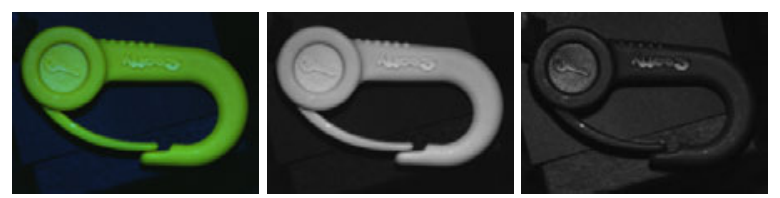

(a) Fluorescent component separated through bispectral measurement: image under near blue light (left), fluorescent-only image observed in the green channel (middle), and reflective-component-only images observed in the blue channel of the camera (right).

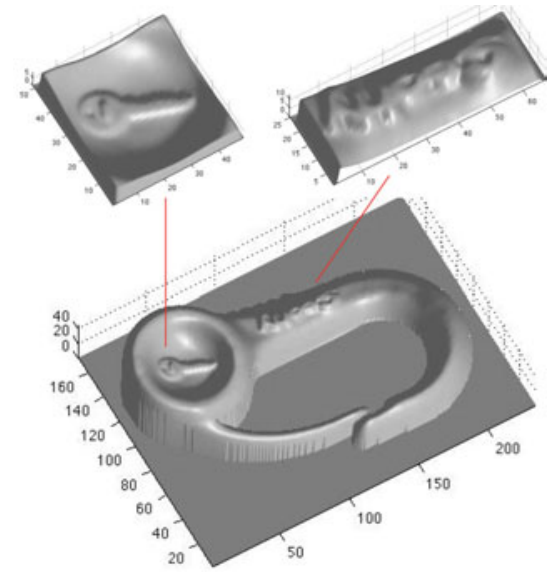

(b) Recovered shape from fluorescence.

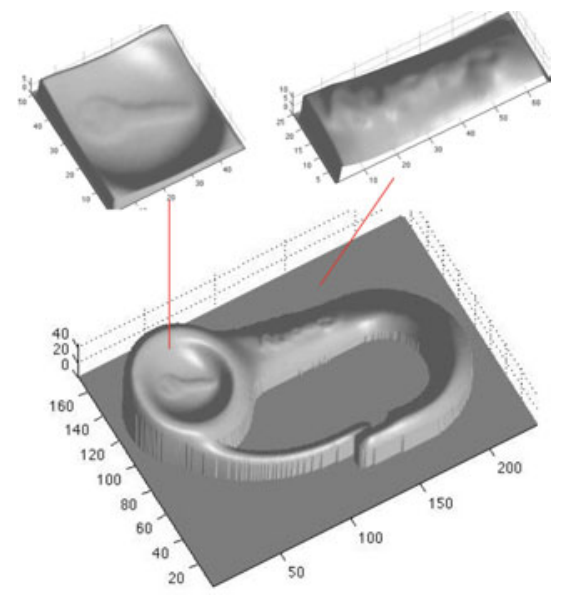

(c) Recovered shape from reflection.

Figure 6. Experimental results for a key holder.

ing reflective components captured under red light in red channel. Compared with the recovered shape from reflective components (Figures 6(c)), we see that the fine details such as letters and a key-shaped object were successfully recovered by our method without suffering from specular reflection and interrefections (Figures 6(b)).

The second object was a ceramic dish composed of ordinary reflective components. This object had strong highlights spread all over its surface (Figure 7(a) left), and this made it difficult to accurately recover its shape using photometric stereo approaches. For instance, if we directly apply the classic photometric stereo to the images of this object,
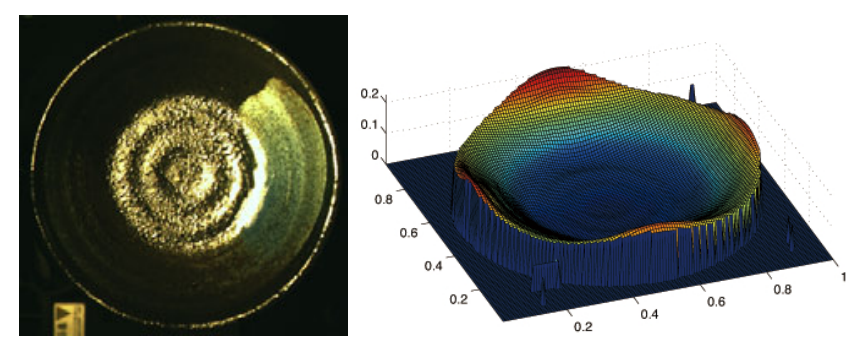

(a) Image of a dish under white light and recovered shape from reflection.
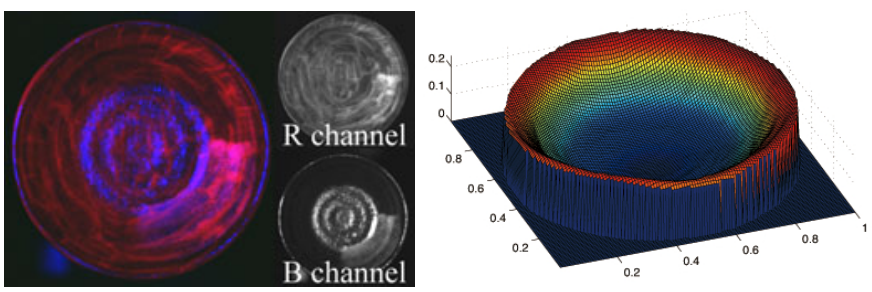

(b) Image of a dish under UV light and recovered shape from fluorescence.

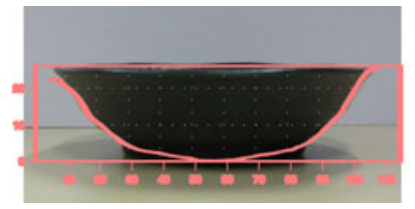

(c) cross-sectional view of recovered shape from fluorescence superimposed into the side view of the dish.

Figure 7. Experimental results for a dish.

the recovered shape is largely affected by its specular reflection (Figure 7(a) right).

To deal with a object with very sharp highlights, we can put special fluorescent paint on it. This paint is invisible under normal lighting, but it appears bright red under strong UV light (Figure 7(b) left). In other words, we can add a fluorescent component to a target object without changing its appearance under normal lighting. In Figure 7(b) left, we see that its non-fluorescent (especially specular reflection) and fluorescent components are separately observed in the blue and red channels of the camera. Figure 7(b) right shows the shape recovered from fluorescence images captured under UV light in the red channel: the cross-sectional view of the recovered shape from fluorescence is superimposed into the side view of the object (Figure 7(c)). We succeeded in obtaining an accurate shape without suffering from specular reflection. This demonstrates the robustness of our bispectral photometric stereo method against specular reflection.

\section{Conclusion}

We studied the appearance of fluorescence emissions under different lighting directions and presented a novel photometric stereo approach that made effective use of fluorescence in shape reconstruction. We showed that there is a 
strong similarity between fluorescence and ideal diffuse reflections and proposed to separate the fluorescence components from ordinary reflection components by using bispectral measurements. The unique wavelength-shifting property of fluorescence enables us to use emission-only images for shape reconstruction without suffering from specular reflection. This is the significant advantage of the fluorescence-based method over previous methods based on reflection. A future direction of this work would be to explore the use of fluorescence in other computer vision algorithms. There are many algorithms in computer vision that assume Lambertian reflectance. We believe that fluorescence is very useful in the sense that it appears like an ideal diffuse surface whose color is not affected by the color of the illumination, and we can easily exclude specular reflection from the observation.

\section{References}

[1] M. Alterman, Y. Schechner, and A. Weiss. Multiplexed fluorescence unmixing. In Proc. IEEE International Conference on Computational Photography, pages 1-8, 2010.

[2] K. Barnard. Color constancy with fluorescent surfaces. pages 257-261, 1999.

[3] K. Barnard, V. Cardei, and B. Funt. A comparison of computational color constancy algorithms. IEEE Transactions on Image Processing, 11(9):972-996, 2002.

[4] S. Barsky and M. Petrou. The 4-source photometric stereo technique for three-dimensional surfaces in the presence of highlights and shadows. 25(10):1239-1252, 2003.

[5] R. Basri, D. Jacobs, and I. Kemelmacher. Photometric stereo with general, unknown lighting. Int. J. Comput. Vision, 72:239-257, 2007.

[6] P. N. Belhumeur, D. J. Kriegman, and A. L. Yuille. The bas-relief ambiguity. Int'l J. Computer Vision, 35(1):33-44, 1999.

[7] M. K. Chandraker, F. Kahl, and D. J. Kriegman. Reflections on the generalized bas-relief ambiguity. In Proc. IEEE Conf. Computer Vision and Pattern Recognition, pages I-788-795, 2005.

[8] E. N. Coleman and R. Jain. Obtaining 3-dimensional hape of textured and specular surfaces using four-source photometry. Computer Vision, Graphics, and Image Processing, 18(4):309-328, 1982.

[9] R. Donaldson. Spectrophotometry of fluorescent pigments. British Journal of Applied Physics, 5(6):210-214, 1954.

[10] P. Emmel and R. D. Hersch. Spectral colour prediction model for a transparent fluorescent ink on paper. In Proc. the Color Imaging Conference: Color Science, Systems, and Applications. Society for Imaging Science and Technology, 1998.

[11] A. S. Georghiades. Incorporating the torrance and sparrow model of reflectance in uncalibrated photometric stereo. In Proc. Int'l. Conf. Computer Vision, pages 816-825, 2003.

[12] A. Glassner. A model for fluorescence and phosphorescence. In Proc. the Eurographics Workshop on Rendering, pages 57-68, 1994.
[13] H. Hayakawa. Photometric stereo under a light-source with arbitrary motion. J. Optical Soc. Am. A, 11(11):3079-3089, 1994.

[14] M. B. Hullin, M. Fuchs, I. Ihrke, H.-P. Seidel, and H. P. A. Lensch. Fluorescent immersion range scanning. ACM Trans. Graph. (Proc. SIGGRAPH 2008), 27(3):87:1-87:10, 2008.

[15] M. B. Hullin, J. Hanika, B. Ajdin, H.-P. Seidel, J. Kautz, and H. P. A. Lensch. Acquisition and analysis of bispectral bidirectional reflectance and reradiation distribution functions. ACM Trans. Graph., 29(4):97:1-97:7, 2010.

[16] K. Ikeuchi. Determining surface orientations of specular surfaces by using the photometric stereo method. IEEE Trans. Pattern Analysis and Machince Intelligence, 3(6):661-669, 1981.

[17] G. M. Johnson and M. D. Fairchild. Full-spectral color calculations in realistic image synthesis. IEEE Computer Graphics and Applications, 19:47-53, 1999.

[18] M. Liao, X. Huang, and R. Yang. Interreflections removal for photometric stereo by using spectrum-dependent albedo. In Proc. IEEE Conf. Computer Vision and Pattern Recognition, 2011.

[19] T. Nakajima and S. Tominaga. Spectral reflectance estimation of fluorescent materials by using camera images. In Proc. Color Science Association of Japan, pages $74-75$, 2010.

[20] S. Nayar, K. Ikeuchi, and T. Kanade. Shape from interreflections. International Journal on Computer Vision, 6(3):173195, 1991.

[21] S. Nayar, G. Krishnan, M. D. Grossberg, and R. Raskar. Fast Separation of Direct and Global Components of a Scene using High Frequency Illumination. ACM Trans. on Graphics (also Proc. of ACM SIGGRAPH), 2006.

[22] S. K. Nayar, K. Ikeuchi, and T. Kanade. Determining shape and reflectance of hybrid surfaces by photometric sampling. IEEE Trans. Robotics and Automation, 6(4):418-443, 1990.

[23] M. Oren and S. Nayar. Generalization of the lambertian model and implications for machine vision. Int'l J. Computer Vision, 14(3):227-251, 1995.

[24] A. Springsteen. Introduction to measurement of color of fluorescent materials. Analytica Chimica Acta, 380(2-3):183 192, 1999.

[25] A. Wilkie, R. Tobler, and W. Purgathofer. A reflectance model for diffuse fluorescent surfaces. In Proc. Graphite 2006, pages 321-328, 2006.

[26] L. Wolff, S. Shafer, and G. E. Healey. Physics-based vision: Principals and Practice, Shape Recovery. Jones and Bartlett., 1992.

[27] R. J. Woodham. Analysing images of curved surfaces. Artif. Intell., 17(1-3):117-140, 1981.

[28] C. Zhang and I. Sato. Separating reflective and fluorescent components of an image. In Proc. Computer Vision and Pattern Recognition., 2011.

[29] T. Zickler, P. N. Belhumeur, and D. J. Kriegman. Helmholtz stereopsis: Exploiting reciprocity for surface reconstruction. International Journal of Computer Vision, 49(2/3):215-227, 2002. 